\title{
Comparison of Probiotic LactobacillusGR-1 and RC-14 with Metronidazole Oral Tablet for Treating Concurrent Bacterial and Trichomonial Vaginitis : A Blind, Randomized, Clinical Trial
}

\author{
Mina Taheri ${ }^{1}$, Khadijeh Abdali ${ }^{1}$, Sedigheh Amooee ${ }^{2}$ and Mehrab Sayadi ${ }^{3}$ \\ ${ }^{1}$ Department of Midwifery, School of Nursing and Midwifery, \\ Shiraz University of Medical Sciences, Shiraz, Iran. \\ ${ }^{2}$ Department of Obstetrics \& Gynecology,School of Medicine, \\ Shiraz University of Medical Science, Shiraz, Iran. \\ ${ }^{3}$ Statistic Counselor, Research Center for Social Determination of Health, \\ Shiraz University of Medical Science,Shiraz,Iran.
}

http://dx.doi.org/10.13005/bbra/2006

(Received: 02 December 2015; accepted: 14 January 2016)

\begin{abstract}
Comparison of the effects of oral Metronidazole and vaginal probiotic capsule in the women suffering from concurrent bacterial and trichomonial vaginitis. A total of 76 women with concurrent bacterial and trichomonial vaginitis were randomly divided into 2 groups.Group A was treated with a twice daily dose of $500 \mathrm{mg}$ oral Metronidazole for 7 days.Group B was treated by vaginal probiotic capsule containing lactobacillus rhamnosus GR-1 and lactobacillus reuteri RC-14 once a day for 10 days.Clinical signs, gram stain, $\mathrm{PH}$ and wet smear were measured in both groups before and after the intervention. The data were analyzed using the SPSS statistical software (v. 16) and McNemar and Chi-square tests. No significant difference was observed between the two groups regarding the clinical signs on the $8^{\text {th }}$ and the $11^{\text {th }}$ days $(P>0.05)$. Also, a significant difference was found between the two groups regarding the wet smear results $(\mathrm{P}<0.05)$. After the follow-up, a significantly higher number of women receiving the probiotic capsule had normal vaginal flora and desirable vaginal PH compared to the Metronidazole group $(\mathrm{P}<0.05)$. Application of vaginal probiotic capsule leads to the stability of the vaginal ecosystem and prevents the growth of the pathogenic bacteria as well as the other pathogens.
\end{abstract}

Key words: Oral Metronidazole, Vaginal probiotic capsule, Concurrent bacterial and trichomonial vaginitis

In 1933, Molher and Brown suggested that lactobacilli replacement therapy could be effective in prevention and treatment of various urogenital infections ${ }^{1}$. After that, a large number of researchers examined the metabolic and biochemical contents of various lactobacillus species for effective colonization of vaginal ecosystem and treatment of vaginitis as well as Vaginosis ${ }^{2}$.

\footnotetext{
* To whom all correspondence should be addressed. Tel: +987136474252-8; Fax: +987136474250; E-mail: abdali_kh@yahoo.com
}

Trichomonial vaginitis is usually accompanied by bacterial vaginosis. In fact, bacterial vaginosis can be simultaneously diagnosed in $60 \%$ of the patients with trichomonial vaginitis $^{3}$. In general, where lactobacillus is dominant, bacteria and parasites, such as Trichomonas, are not abundant. Also, in lack of lactobacillus, a considerable increase can be observed in infections, such as bacterial vaginosis and vaginitis, and sexually transmitted diseases, including gonorrhea, Chlamydia, syphilis, Trichomoniasis, HIV, and HPV ${ }^{4}$.

The present prospective, single-blind, randomized, clinical trial was conducted on 76 
women in the reproductive age in order to compare the effect of vaginal probiotic capsule and Metronidazole oral tablet in treatment of concurrent bacterial and trichomonial vaginitis.

\section{MATERIALSAND METHODS}

The present single-blind, randomized, clinical trial was conducted on 100 women with concurrent bacterial and trichomonial vaginitis who had referred to outpatient gynecology clinics of Shiraz University of Medical Sciences, Shiraz, Iran between December 2011 and July 2012 and had the inclusion criteria of the study.

Diagnosis of BV was defined as meeting at least three items of the Amsel criteria (clue cells, off-white thin homogeneous vaginal discharge, and vaginal $\mathrm{pH} \geq 4.5$ ) and gram stain. In addition, Trichomonas diagnosis was defined as positive wet smear.

The exclusion criteria of the study were pregnancy, lactation, being younger than 18 or older than 55, presence of other urogenital infections, cigarette smoking, menstruation at time of screening, hypersensitivity to Metronidazole, and being considered unhealthy based on the clinical diagnosis but having negative test results.

This trial was approved by the Ethical Review Board and the Dean of Faculty, the Deputy of Educational Affairs, Shiraz University of Medical Science, Shiraz, Iran. Written informed consents were obtained from all of the women at the beginning of the study. The trial was registered at irct.ir IRCT138711201548N3.

The objectives of this study were the comparison of the ratios between 2 study groups before and after treatment. We calculated that 40 women were needed in each of the 2 study groups with 38 per group completing the study, to have $80 \%$ power to detect a $28 \%$ difference in ratios between the two groups ( $43 \%$ vs. $15 \%$ ) at the statistical significance level of $\alpha<0.05$.

A total of 76 women who satisfied the inclusion criteria were enrolled into the study and randomly allocated into 2 groups by blocking design. The women in group A (37 women) were treated with a twice daily dose of $500 \mathrm{mg}$ oral metronidazole (Metronidazole; Alborz Darou Pharmaceutical Co, Tehran, Iran) for 7 days. On the other hand, the women in group B (39 women) were treated with the vaginal application of one gelatin capsule containing Lactobacillus rhamnosus GR-1 and Lactobacillus reuteri RC-14 (1billion CFU) (Fembio, Zydus healthcare company, Majhitar, Rangpo, East Sikkim, India) once a day for 10 days.

Follow-up was performed immediately after the intervention; i.e., on day 8 in the Metronidazole group and day 11 in the probiotic capsule group. It should be mentioned that clinical examination and $\mathrm{PH}$ measurement were performed by the interventionist who collected the samples, as well.

Microscopic examination of the wet mounts and gram stains were carried out blindly by a skilled laboratory technician.

Healing was defined as the absence of $\mathrm{BV}$ according to the Amsel criteria, microscopic evidence of a greater number of lactobacilli than the other bacterial flora, and absence of motile flagellate on the wet mount.

On a scheduled visit, after inspection of the vulva and vestibule during the physical examination, an unmoistened sterile speculum was inserted into the vagina so that the vaginal walls, fornices, and the cervix could be evaluated for eventual erythema, color, and viscosity of the discharge. The PH value of the vaginal walls and the lateral Fornices was measured by colorimetric paper with 14 comparison colors for $\mathrm{PH}$ values ranging from 1 to 14 (Universal Indicator Paper, Hangzhou Special Paper Industry Co.,LTD, Hangzhou municipality, China). It should be noted that the vaginal samples were collected from the lateral fornices by a cotton spatula. Two swabs were taken from the vaginal wall. One sample was immediately put in 1cc warm saline in a test tube and sent to the laboratory for diagnosis of trichomonial infection based on the wet smear (presence of motile flagellates on wet mount). Yet, the other sample was expanded on the slide and after being dried on the slide, it was sent to the laboratory for gram staining in order to diagnose bacterial vaginosis.

When the symptoms and signs of mixed (bacterial and trichomonial) vaginitis were present during the follow up, the participant were (re-) treated with Metronidazole.

At the end of the study, the data were collected and analyzed using Chi-square (Fisher 
exact) and McNemar statistical tests at $\alpha$ level of 0.05 (the confidence intervals were 95\%). All the statistical analyses were performed through the SPSS statistical software, version 16.0 (SPSS, Chicago, IL, USA).

\section{RESULTS}

Among the 100 screened patients, 76 participants (76\%) were eligible, 37 of whom were randomized to receive Metronidazole and 39 to receive probiotic. After randomization, no one was excluded from the study.Before the treatment, no significant difference was found between the two groups regarding their clinical signs, gram staining, and wet smear (Table 1-3).

Besides, PH values between 4.5 and 5.5 were most frequent in both study groups before the treatment (Table 4).

After treatment, a significant difference in improvement of clinical sign was observed in both groups (Table 1). This means that both drugs have similar effects in improving clinical signs.

A statistically significant difference was also found between the two groups regarding the rate of improvement based on the gram stain results (Table 2). Considering wet smear, although a significant difference was observed in each group's status before and after the treatment, the frequency percentage of negative wet smear in the probiotic group was less than that of the metronidazole group (Table 3).

After the treatment, however, $\mathrm{PH}$ between 4.5 and 5.5 (73\%) in the Metronidazole group and

Table 1. Clinical signs and symptoms ${ }^{\mathrm{a}}$

\begin{tabular}{llllll}
\hline Medication & Features & & \multicolumn{2}{c}{ Treatment } & \multicolumn{2}{c}{ pvalue $^{\text {b }}$} \\
\cline { 5 - 6 } & & & $\begin{array}{l}\text { After } \\
\text { Number (\%) }\end{array}$ & Number(\%) & \\
\hline Oral metronidazole & Vaginal discharge & Yes & $36(97.30)$ & $6(16.20)$ & $<0.001$ \\
(Group A)(n=37) & Malodor discharge & Yes & $32(86.50)$ & $6(16.20)$ & $<0.001$ \\
& Itching & Yes & $26(70.30)$ & $6(16.20)$ & $<0.001$ \\
& Dyspareunia & Yes & $21(56.80)$ & $8(21.06)$ & 0.004 \\
& Irritation & Yes & $14(37.80)$ & $5(13.05)$ & $<0.001$ \\
& Dysuria & Yes & $8(21.60)$ & $0(0)$ & $<0.001$ \\
Probiotic capsule & Abdominal pain & Yes & $22(59.50)$ & $8(21.60)$ & $<0.001$ \\
(Group B) & Vaginal discharge & Yes & $39(100)$ & $5(12.80)$ & $<0.001$ \\
(n=39) & Malodor discharge & Yes & $37(94.90)$ & $0(0)$ & $<0.001$ \\
& Itching & Yes & $24(61.5)$ & $7(17.90)$ & $<0.001$ \\
& Dyspareunia & Yes & $21(53.80)$ & $5(12.80)$ & $<0.001$ \\
& Irritation & Yes & $16(41)$ & $4(10.3)$ & $<0.001$ \\
& Dysuria & Yes & $6(15.4)$ & $0(0)$ & $<0.001$ \\
& Abdominal pain & Yes & $25(61.4)$ & $6(15.40)$ & $<0.001$ \\
\hline
\end{tabular}

${ }^{a}$ Values are given as number (percentage)

${ }^{\mathrm{b}}$ by McNemar test

Table 2. Number of patients with normal vaginal ecosystems before and after treatment in both $\mathrm{A}$ and $\mathrm{B}$ groups

\begin{tabular}{lcc}
\hline & $\begin{array}{c}\text { No.(\%) of patient with negative wet smear } \\
\text { Group (A)Oral } \\
\text { capsulesN=39 }\end{array}$ & $\begin{array}{c}\text { Group (B)Probiotic } \\
\text { MetronidazoleN=37 }\end{array}$ \\
\hline Before treatment & $0(0)$ & $0(0)$ \\
After treatment & $33(89.2)$ & $3(7.2)$ \\
P value $^{\mathrm{a}}$ & $<0.001$ & $<0.001$ \\
\hline
\end{tabular}

a by McNemar test 
$\mathrm{PH}<4.5$ (46.2\%) in the probiotic group were most frequent and a significant decrease was observed in $\mathrm{PH}$ of the probiotic group compared to the Metronidazole group (Table 4).

In this study, no adverse events were reported by the women in the probiotics groups compared to those receiving Metronidazole (19 gastro-intestinal, 18 dizziness). These events were rated as mild to moderate, no severe events occurred, and treatment was not interrupted on any occasion.

Table 3. Number of patients with negative wet smear result before and after treatment in both $\mathrm{A}$ and $\mathrm{B}$ groups

\begin{tabular}{lcc}
\hline & $\begin{array}{c}\text { No.(\%) of patient with normal vaginal ecosystems } \\
\text { Group (A)Oral } \\
\text { capsulesN=39 }\end{array}$ & $\begin{array}{c}\text { Group (B)Probiotic } \\
\text { MetronidazoleN=37 }\end{array}$ \\
\hline Before treatment & $0(0)$ & $0(0)$ \\
After treatment & $20(54.1)$ & $34(87.2)$ \\
P value $^{\text {a }}$ & $<0.001$ & $<0.001$ \\
\hline
\end{tabular}

a by McNemar test

Table 4. Vaginal PH before and after treatment

\begin{tabular}{|c|c|c|c|c|c|c|}
\hline \multirow[t]{2}{*}{ PHGroup } & \multicolumn{3}{|c|}{ Before } & \multicolumn{3}{|c|}{ After } \\
\hline & $<4.5$ & $4.5-5.5$ & $>5.5$ & $<4.5$ & $4.5-5.5$ & $>5.5$ \\
\hline Metronidazole (Group A , N=36), n(\%) & $0(0.00)$ & $21(58.80)$ & $16(43.20)$ & $2(5.40)$ & $27(73.00)$ & $8(21.60)$ \\
\hline Probiotic (Group B , N=39), n(\%) & $0(0.00)$ & $25(64.10)$ & 14(35.90) & $18(46.20)$ & 21(53.80) & $0(0.00)$ \\
\hline P-value ${ }^{\mathrm{a}}$ & 0.639 & 0.001 & & & & \\
\hline
\end{tabular}

a: fisher exact test was used

\section{DISCUSSION}

Recent studies have shown that probiotic lactobacillus organisms can be effectively used in vaginal colonization and interfere with the urogenital pathogens ${ }^{5}$. The results of the present study also showed that 10-day treatment with vaginal probiotic lactobacilli can be effective in treatment of concurrent bacterial and trichomonial vaginitis as well as maintenance of vaginal lactobacilli. The positive results of this trial might be due to the type of the lactobacilli utilized in the probiotic capsules or the volume of the lactobacilli. In fact, the dose utilized in this study was 10 times more than the volume recommended for restoration and maintenance of urogenital system's normal flora. Although this mechanism has not been clearly identified yet, this dose probably affects the probiotic-microbiological load of the vagina and colonizes the vagina with sufficient concentrations of the lactobacilli and, consequently, destroys or prevents the growth of the pathogenic bacteria and stops the attachment of gardnerella vaginalis ${ }^{6}$. In the current study, the major advantage of treatment with lactobacilli was improvement of clinical sign similar to Metronidazole group, e.g. reduction of malodor vaginal discharges which is the first complaint and mental sign of the women with concurrent bacterial and trichomonial vaginitis.

Comparison of the treatment outcomes in the two groups based on gram staining revealed a statistically significant difference in both groups compared to before the intervention $(\mathrm{P}<0.05)$. These results were in agreement with those of the studies conducted by Wang Ya et al., ${ }^{7}$ and Mastromarino et al., ${ }^{8}$

Although the results of wet smear test were statistically significant in the probiotoc group, a fewer number of patients in this group had negative wet smear after the treatment compared to the Metronidazole group ( $7.2 \%$ and $89.2 \%$, 
respectively).Reports suggest that lactobacilli counts are greatly reduced in infection with Trichomonas vaginalis and lactobacillus suppositories are used in treatment of Trichomoniasis from thirty years ${ }^{9}$.Also Trichomonas will not grow well in the presence of high concentrations of lactobacilli ${ }^{10}$.

Comparison of the changes in vaginal $\mathrm{PH}$ in the two groups before and after the intervention showed that vaginal probiotic capsule was more effective than Metronidazole oral tablet in improving the vaginal $\mathrm{PH}$. After the treatment, $46.2 \%$ of the patients in the probiotic group had $\mathrm{PH}<4.5$, while this $\mathrm{PH}$ level was only observed in $5.4 \%$ of the women in the Metronidazole group.

Vaginal epithelial cells are the most important place in the initial contact of trichomonas vaginitis $^{11}$. In human infection, parasite adhesion, invasion, and retention are of great importance. Although lactobacillus is effective in adhesion of the parasite to the vaginal epithelial cells at the primary stages of infection, the parasite can be eliminated by increasing the number of the bacteria and decreasing the vaginal $\mathrm{PH}$, particularly in the drug-resistant patients. This parasite adheres to the vaginal epithelial cells through adhesion proteins $^{12}$ whose activation depends on time, temperature, and $\mathrm{pH}^{13}$. In case the number of lactobacilli is increased in the vagina, the materials secreted from the bacteria and reduction of $\mathrm{PH}$ can play a critical role in removing the parasite.

Overall, vaginal application of lactobacillus rhamnosus and GR-1 lactobacillus, as a beneficent complementary therapy, can be used in management of the urinary system infections ${ }^{14}$. Thus, specific clinical trials are required for supporting the expansion of probiotic treatments to a wide range of urogenital infections as well as sexually transmitted diseases. Furthermore, it seems that investigation of the patients' microbiological and immunological parameters before, during, and after the treatment with Metronidazole and probiotics can help in better understanding of the probiotics efficiency in eliminating the clinical signs.

\section{ACKNOWLEDGMENTS}

The present article was extracted from the thesis written by Mina Taheri and was financially supported by Shiraz University of Medical Sciences grants No. 90-5809. The authors have no conflicts of interest to disclose.

\section{REFERENCES}

1. Molher RW, Brown CP. Doderlein's bacillus in the treatment of vaginitis. Am J Obstet Gynecol 1933;25: 718-23.

2. Marcone V, Rocca G, Lichtner M, Calzolari E: Long-term vaginal administration of Lactobacillus rhamnosus as a complementary approach to management of bacterial vaginosis. Int J Gynecol Obstet 2010;110(3): 223-26.

3. Soper DE. Genitourinary Infections and Sexually Transmitted Diseases .In: Berek, Jonathan S. Berek \& Novak’s Gynecology. Lippincott Williams \& Wilkins.2007; 810-10.

4. Donders GGG: Definition and classification of abnormal vaginal flora. Best Pract Res Clin Obstet Gynaecol 2007;21(3): 355-73.

5. Anukam KC, Osazuwa E, Osemene GI, Ehigiagbe F, Bruce AW, Reid G: Clinical study comparing probiotic Lactobacillus GR-1 and RC-14 with metronidazole vaginal gel to treat symptomatic bacterial vaginosis. Microbes Infect 2006; 8(12-13): 2772-76.

6. Falagas ME, Betsi GI, Athanasiou S: Probiotics for the treatment of women with bacterial vaginosis. Clin Microbiol Infect 2007;13(7): 65764.

7. Ya W, Reifer C, Miller LE: Efficacy of vaginal probiotic capsules for recurrent bacterial vaginosis: a double-blind, randomized, placebocontrolled study. Am J Obstet Gynecol 2010; 203(2): 120.e121-120.e126.

8. Mastromarino P, Macchia S, Meggiorini L, Trinchieri V, Mosca L, Perluigi M, et al.: Effectiveness of Lactobacillus-containing vaginal tablets in the treatment of symptomatic bacterial vaginosis. Clin Microbiol Infect 2009;15(1): 6774.

9. Hillier SL, Krohn ME, Klebanoff SJ, Eschenbach DA: The relationship of hydrogen peroxideproducing lactobacilli to bacterial vaginosis and genital microflora in pregnant women. Int $J$ Gynecol Obstet 1992;39(2): 152-152.

10. Pi W, Ryu J-S, Roh J: Lactobacillus acidophilus Contributes to a Healthy Environment for Vaginal Epithelial Cells. Korean J Parasitol 2011;49(3): 295-98.

11. Torok MR, Miller WC, Hobbs MM, MacDonald PDM, Leone PA, Schwebke JR, et al.: The Association between Trichomonas vaginalis Infection and Level of Vaginal 
Lactobacilli, in Nonpregnant Women. J Infect Dis 2007;196(7): 1102-07.

12. Arroyo R, Engbring J, Alderete JF: Molecular basis of host epithelial cell recognition by Trichomonas vaginalis. Mol Microbiol 1992; 6(7): 853-862.

13. Alderete JF, Demeœ P, Gombosova A, Valent
M, Fabusová M, Jánoska A, et al.: Specific parasitism of purified vaginal epithelial cells by Trichomonas vaginalis. Infect Immun 1988; 56(10): 2558-2562.

14. Reid G: Probiotic agents to protect the urogenital tract against infection. Am J Clin Nutr 2001;73(2 Suppl): 437S-443S. 